\title{
Exact solution of the envelope equations for a matched quadrupole-focused beam in the zero space-charge limit
}

\author{
O. A. Anderson \\ LBNL, Berkeley, California 94720, USA \\ L. L. LoDestro \\ LLNL, Livermore, California 94551, USA
}

(Received 4 November 2008; published 6 May 2009)

\begin{abstract}
The Kapchinskij-Vladimirskij equations are widely used to study the evolution of the beam envelopes in a periodic system of quadrupole focusing cells. In this paper, we analyze the case of a matched beam. Our model is analogous to that used by Courant and Snyder [E. D. Courant and H. S. Snyder, Ann. Phys. (Paris) 3, 1 (1958)], who obtained a first-order approximate solution for a synchrotron. Here, we treat a linear machine and obtain an exact solution. The model uses a full occupancy, piecewise-constant focusing function and neglects space charge. There are solutions in an infinite number of bands as the focus strength is increased. All these bands are stable. Our explicit results for the phase advance $\sigma$ and the envelopes $a(z)$ and $b(z)$ are exact for all phase advances except multiples of $180^{\circ}$, where the behavior is singular. We find that the peak envelope size is minimized for $\sigma \sim 81^{\circ}$. Actual operation in the higher bands would require very large, very accurate field strengths and would produce significantly larger envelope excursions. If such operation were found to be feasible, there would be interesting applications which we discuss.
\end{abstract}

DOI: 10.1103/PhysRevSTAB.12.054201

PACS numbers: 41.85.Ja, 52.59.Wd

\section{INTRODUCTION}

The Kapchinskij-Vladimirskij (KV) equations [1] describe the evolution of the beam envelopes in a periodic system of quadrupole focusing cells and are widely used to help predict the performance of such systems, using various methods of solution. Numerical solutions of the KV equations give useful results in many cases, but with very strong focusing fields there can be difficulties with numerical convergence [2] and with extreme sensitivity to input parameters. Analytic solutions are useful for the insight they provide into qualitative features and trends. There have been numerous papers presenting approximate analytic solutions giving various degrees of accuracy. The simplest, known as the smooth approximation [3,4], is useful for lower field strengths but in some forms can give the misleading impression that the average beam size continues to decrease indefinitely as the focus fields increase. Higher approximations [5,6] extend the range of accuracy but all approximation schemes eventually fail at sufficiently strong fields. Partly due to these limitations, the strong-field regime has not been thoroughly investigated. A primary motivation for the present paper is to obtain accurate solutions for high field strengths and determine whether there are strong-field regimes that might have practical applications.

In this paper, we derive an exact solution for matched beam envelopes (periodic with the period of the focusing lattice) for arbitrary field strength. We treat a problem examined by Courant and Snyder in their classic paper [7], except that we assume a linear rather than circular machine. They discussed the case in which the focus and defocus sections each had uniform focusing strength with no intervening gaps. They called this the CLS configuration [8]. Their model neglected space charge, as we do in the present paper. Although space charge is no longer negligible in typical modern devices, our exact solution for this case does provide insight into the general behavior of alternating-gradient systems and can provide a starting point for analysis of other focusing models and for the case of finite space charge. [Exact results have also been obtained-for the thin-lens ( $\delta$-function) focusing model-by Takayama in the zero space-charge limit [9] and by Lund and Bukh in their complete analysis of the zero-emittance case [10].]

Courant and Snyder used the CLS model for a synchrotron, obtaining approximate solutions. We show that the analogous model for a linear machine is exactly solvable and explore the consequences. We will refer to our model (piecewise-constant focus, negligible space charge, straight rather than circular beam axis) as the CSS model [11]. For this model: (1) We find that solutions for the envelopes $a(z)$ and $b(z)$ exist in an infinite number of bands and that these coincide with bands of single-particle stability. (2) We obtain a well-defined expression for the phase advance $\sigma$ as a function of focusing strength that applies to all bands. All values of $\sigma$ are theoretically possible except exact multiples of $180^{\circ}$. (3) The peak beam radius is minimized when $\sigma=80.57^{\circ}$ and begins to increase rapidly above $\sigma \sim 100^{\circ}$. Stable higher bands in 


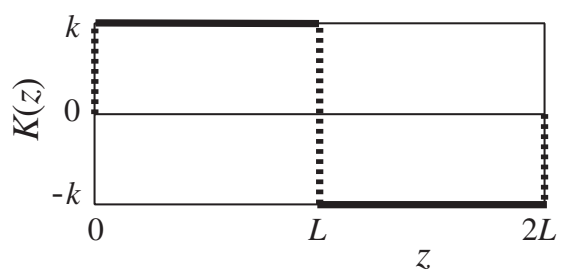

FIG. 1. CSS focusing model for the $x z$ plane, shown for a single cell of the periodic lattice. The $y z$-plane fields are the same but shifted by the distance $L$.

quadrupole channels have been previously mentioned in, for example, Refs. [10,12]. The higher bands give larger beam excursions in spite of greatly increased focusing fields [10]. Although the minimum radius is reduced, the average radius and peak radius are increased. It is the peak radius that is significant for focusing lattices of limited aperture. For such systems, in the emittance-dominated regime, we find no advantage in increasing the focusing strength beyond values that give $\sigma$ larger than $81^{\circ}-$ except, perhaps, for special applications, such as the possibility of extreme beam compression discussed in Secs. VIII and X.

\section{FOCUSING MODEL}

We assume a quadrupole-symmetric focusing function $K(z)$ that is piecewise constant with values $\pm k$ and periodic over a lattice with period $2 L$. This model is described by Eqs. (1) and Fig. 1 for a single lattice cell in the $x z$ plane:

$$
K(z) \equiv \begin{cases}+k & \text { for } 0<z<L \\ -k & \text { for } L<z<2 L\end{cases}
$$

In a lattice with quadrupole symmetry, the $x z$ plane and $y z$-plane envelopes $a(z)$ and $b(z)$ for a matched beam are identical except for a shift of length $L$ [6]. Thus, it is only necessary to analyze the dynamics in one of the planes; we choose the $x z$ plane here.

\section{SINGLE-PARTICLE STABILITY}

In the absence of space charge, the transverse position $x(z)$ of a particle is determined by

$$
x^{\prime \prime}(z)+K(z) x(z)=0 .
$$

The stability of the single-particle orbit is easily found from the period-transfer matrix $M[7,13]$ and is given by $|\operatorname{Tr}(M)|<2$. With $K(z)$ defined by Eq. (1), this yields

$$
|\cos \sqrt{k} L|<\operatorname{sech} \sqrt{k} L \text {. }
$$

Figure 2 shows that there are multiple bands of stable solutions over increasingly narrow ranges of $\sqrt{k} L$.

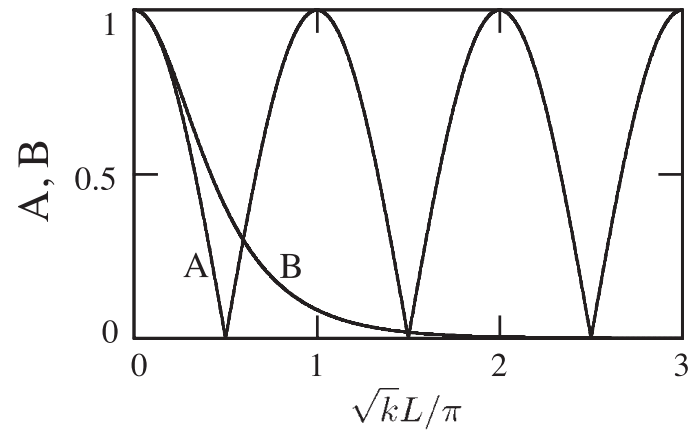

FIG. 2. $\mathrm{A}=|\cos \sqrt{k} L| ; \quad \mathrm{B}=\operatorname{sech} \sqrt{k} L$. Stable solutions of Eq. (2) exist in the regions where curve A lies below curve B.

\section{DERIVATION OF THE EXACT SOLUTION OF THE ENVELOPE EQUATIONS}

In the CSS model, which neglects space charge, the $x z$-plane envelope $a(z)$ of a beam with emittance $\in$ obeys [13]

$$
a(z)^{\prime \prime}+K(z) a-\frac{\epsilon^{2}}{a^{3}}=0
$$

with $K(z)$ from Eq. (1). The boundary conditions are periodic for a matched beam.

Multiplying Eq. (4) by $2 a^{\prime}$ and integrating, one obtains

$$
a^{\prime 2}+K(z) a^{2}+\frac{\in^{2}}{a^{2}}=C
$$

Then, multiplying Eq. (5) by $a^{2}$ yields

$$
d z=\frac{d a^{2}}{2 \sqrt{-K(z) a^{4}+C a^{2}-\epsilon^{2}}} .
$$

Any standard table of integrals shows that the solution for $a^{2}(z)$ will involve trigonometric or hyperbolic functions, depending on the sign of $K$. We will find the appropriate constants by using trial solutions.

We define separate functions for the focus $(K=+k)$ and defocus $(K=-k)$ sections:

$$
a(z)= \begin{cases}a_{+}(z) & \text { for } 0<z<L \\ a_{-}(z) & \text { for } L<z<2 L\end{cases}
$$

and choose trial solutions that satisfy the symmetry of $K(z)$ :

$$
\begin{aligned}
& \frac{\sqrt{k}}{\epsilon} a_{+}{ }^{2}(z)=\varphi+F \cos [\lambda(z-L / 2)], \\
& \frac{\sqrt{k}}{\epsilon} a_{-}^{2}(z)=\gamma+G \cosh [\lambda(z-3 L / 2)],
\end{aligned}
$$

where $\lambda, \varphi, F, \gamma$, and $G$ are constants. They will be determined by substituting Eqs. (8) into Eq. (4) and invoking continuity of values and derivatives of $a_{+}$and $a_{-}$at the junctions. 
For the focus section, we differentiate Eq. (8a) twice and find after some algebra that

$$
a_{+}^{\prime \prime}=-\frac{\lambda^{2}}{4} a_{+}(z)\left(1+\frac{\in^{2}}{k} \frac{F^{2}-\varphi^{2}}{a_{+}{ }^{4}}\right) .
$$

Comparison with Eq. (4) gives

$$
\lambda=2 \sqrt{k},
$$

the first of the five unknown constants, and

$$
\varphi^{2}=F^{2}+1
$$

For the defocus section, Eq. (10) still applies and we find similarly

$$
\gamma^{2}=G^{2}-1
$$

\section{A. Introduction of the focus-strength parameter $\theta$}

The focus-strength parameter, used throughout this paper, is defined by

$$
\theta \equiv \sqrt{k} L,
$$

and our trial solutions become

$$
\begin{aligned}
& \frac{\sqrt{k}}{\epsilon} a_{+}{ }^{2}(z)=\varphi+F \cos [\theta(2 z / L-1)], \\
& \frac{\sqrt{k}}{\in} a_{-}{ }^{2}(z)=\gamma+G \cosh [\theta(2 z / L-3)] .
\end{aligned}
$$

The first derivatives are

$$
\begin{aligned}
& \frac{\sqrt{k}}{\epsilon} a_{+}{ }^{2}(z)^{\prime}=-(2 \theta / L) F \sin [\theta(2 z / L-1)], \\
& \frac{\sqrt{k}}{\in} a_{-}{ }^{2}(z)^{\prime}=+(2 \theta / L) G \sinh [\theta(2 z / L-3)] .
\end{aligned}
$$

We find it convenient to use the following abbreviations in the remainder of this section:

$$
\begin{aligned}
& s n \equiv \sin \theta, \quad c s \equiv \cos \theta, \\
& s h \equiv \sinh \theta, \quad c h \equiv \cosh \theta .
\end{aligned}
$$

\section{B. Continuity conditions}

The four remaining unknown constants, $\varphi, F, \gamma$, and $G$, are found by invoking continuity of $a$ and $a^{\prime}$ at $z=L$, the boundary between focus and defocus sections. The symmetries built into our trial solutions guarantee periodicity of $a(z)$ with period $2 L$.

Using Eqs. (16) and (17), we equate derivatives at $z=L$ :

$$
G=\rho F,
$$

with the ratio $\rho$ defined by

$$
\rho \equiv s n / s h
$$

We equate ${a_{+}}^{2}(L)$ and ${a_{-}}^{2}(L)$ using Eqs. (14) and (15):

$$
\gamma-\varphi=(c s-\rho c h) F .
$$

For our four unknowns we now have four equations: (11), (12), (19), and (21). The first three yield

$$
\varphi^{2}+\gamma^{2}=\left(1+\rho^{2}\right) F^{2} .
$$

From Eq. (21),

$$
\gamma^{2}+\varphi^{2}-2 \gamma \varphi=(c s-\rho c h)^{2} F^{2} .
$$

Combining Eqs. (22) and (23) yields

$$
\gamma \varphi=F^{2} \rho \text { chcs. }
$$

We square this and use Eqs. (11) and (12). After a little more algebra, we find

$$
\rho^{2}\left(1-c s^{2} c h^{2}\right) F^{4}-\left(1-\rho^{2}\right) F^{2}-1=0 .
$$

The real solutions are $F= \pm\left(\rho^{2} c h^{2}-1\right)^{-1 / 2}$. To determine the sign of $F$, we note from Eqs. (12) and (15) that $G>0$. Then, from Eq. (19), $\operatorname{sign}(F)=\operatorname{sign}(s n)$. We define the function

$$
\mathrm{P}(\theta) \equiv \operatorname{sign}(s n)
$$

and write

$$
F=\frac{\mathrm{P}}{\sqrt{\rho^{2} c h^{2}-1}}=\frac{\mathrm{P} s h}{\sqrt{1-c s^{2} c h^{2}}} .
$$

From Eq. (11), $\varphi=\mathrm{P} \operatorname{sn} c h\left(1-c s^{2} c h^{2}\right)^{-1 / 2}$. The P function is required here since, according to Eq. (14), $\varphi$ is necessarily positive.

\section{Exact solution}

Finally, then, Eqs. (14), (15), (19), and (21) yield the exact solution

$$
\begin{aligned}
& a_{+}{ }^{2}(z)=\in L \frac{s n c h+s h \cos [\theta(2 z / L-1)]}{P \theta \sqrt{1-c s^{2} c h^{2}}}, \\
& a_{-}{ }^{2}(z)=\in L \frac{s h c s+s n \cosh [\theta(2 z / L-3)]}{P \theta \sqrt{1-c s^{2} c h^{2}}},
\end{aligned}
$$

where the functions $s n, c s, s h$, and $c h$, defined in Eq. (18), depend on the field-strength parameter $\theta$.

Figure 3 plots $a(z) / \sqrt{\epsilon L}$ for various focusing strengths $\theta$ within the stable passbands discussed below. Phase advances $\sigma$ (Sec. V) are also shown. Throughout this paper we usually express $\theta$ in radians and $\sigma$ in degrees, the traditional units. However, $\sigma$ is more conveniently kept in radians in Secs. V and VII. 


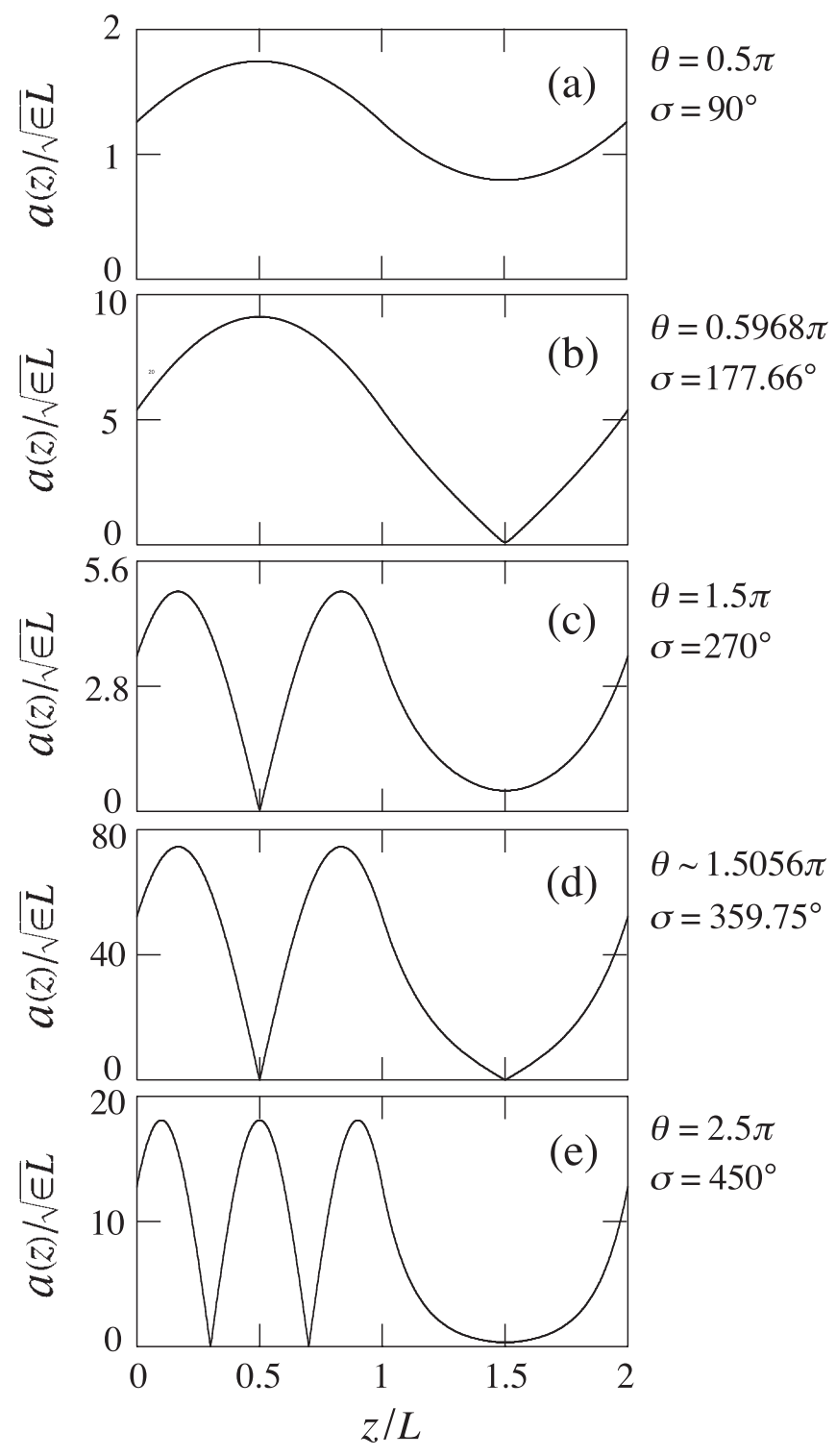

FIG. 3. Plots of Eqs. (28) for various focusing strengths $\theta$ with fixed emittance $\in$. (a) $\theta=0.5 \pi$, midpoint of the first stable band; (b) $\theta=0.5968 \pi$, near band 1 edge; (c) $\theta=1.5 \pi$, the midpoint of band 2; (d) $\theta=1.50561868 \pi$, near band 2 edge; (e) $\theta=2.5 \pi$, band 3 midpoint. (The phase advance $\sigma$, shown for each case, is discussed in Sec. V.) The peak radius is smallest for case (a), where the focus strength is not far from its optimum value $\theta \sim 0.48 \pi$-see Sec. VI. In the even bands, both $a(z)$ and $b(z)$ have minima at the points $z=L / 2$ and $3 L / 2$. Thus, at these points there are large reductions in beam area (see Sec. VIII) for case (c) and huge reductions for case (d). [But note the required field accuracy is four figures for (c) and nine figures for (d); see also Sec. X, where other limitations on beam compression are discussed.]

Equations (28) have real solutions (passbands) when their denominators are real. The existence criterion is

$$
c s^{2} c h^{2}<1
$$

the same as the single-particle stability criterion, Eq. (3).

\section{PHASE ADVANCE IN THE STABLE BANDS}

Equation (28), the matched solution of Eq. (4), has stable bands which surround the points where $\cos \theta=0$. We call these the midpoints $\theta_{n}$ for the focus-strength parameter:

$$
\theta_{n} \equiv\left(n-\frac{1}{2}\right) \pi \quad n=1,2,3 \ldots,
$$

where $n$ is the passband number.

From Ref. [7], the full-period phase advance $\sigma(\theta)$ for the CSS case is given by (assuming $|c s c h|<1$ )

$$
\cos \sigma=\frac{1}{2} \operatorname{Tr} M=\cos \theta \cosh \theta .
$$

(Note that $\cos \sigma=0$ when $\cos \theta=0$, so that $\sigma=\theta$ when $\theta=\theta_{n}$.)

Solving for $\sigma$ by writing $\sigma(\theta)=\cos ^{-1}(\cos \theta \cosh \theta)$ raises the issue of determining which branch of $\cos ^{-1}$ to use for the higher passbands. To address this problem, we first introduce the deviations $\Delta \theta$ and $\Delta \sigma$, defined by

$$
\theta=\theta_{n}+\Delta \theta ; \quad \sigma=\theta_{n}+\Delta \sigma,
$$

where $-\frac{\pi}{2}<\Delta \sigma<\frac{\pi}{2}$ and where $\Delta \theta$ has a smaller range (extremely small for $n>1$ ). Substitution of Eqs. (32) into Eq. (31) gives

$$
\sin \Delta \sigma=\sin \Delta \theta \cosh \theta
$$

with $|\sin \Delta \theta \cosh \theta|<1$. Then

$$
\sigma(\theta)=\theta_{n}+\sin ^{-1}(\sin \Delta \theta \cosh \theta)
$$

Here, $\sin ^{-1}$ is restricted to its principal value, removing the ambiguity mentioned above. Figure 4 displays $\sigma(\theta)$ for the first two bands.

From Eq. (34) and Fig. 4 we see that, for any band $n, \sigma$ has maximum and minimum values

$$
\sigma_{\max }=n \pi, \quad \sigma_{\min }=(n-1) \pi .
$$

In all passbands, $\sigma$ ranges over $\pi$ radians, so that arbitrary $\sigma$ is possible except for the singular points $\sigma=n \pi$. The
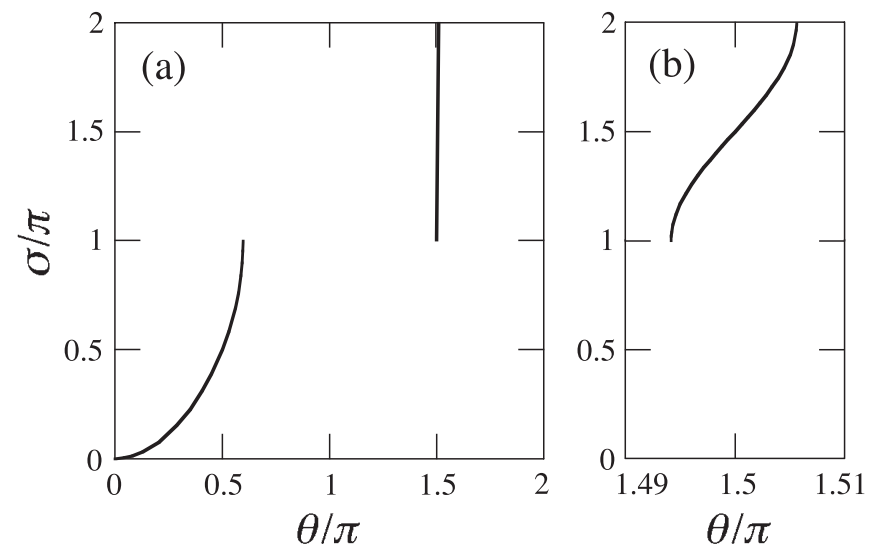

FIG. 4. (a) Phase advance [Eq. (34)] for the first two stable bands. (b) The second band again with the $\theta$ axis magnified. 
required precision of $k$ becomes extreme as these points are approached.

\section{MAXIMUM ENVELOPE EXCURSION}

The peak radius $a_{\max }$ is found from Eq. (28a) by setting the cosine term (containing $z$ ) equal to $\mathrm{P}$, yielding

$$
\frac{a_{\mathrm{max}}^{2}(\theta)}{\in L}=\frac{\mathrm{P} s n c h+s h}{\theta \sqrt{1-c s^{2} c h^{2}}}
$$

Figure 5 illustrates Eq. (36), showing $a_{\max } / \sqrt{\in L}$ as a function of $\theta$ for the first two stable bands. In Fig. 5(a), the peak radius $a_{\max }$ decreases with increasing field strength up to the point where $\theta \sim 0.48 \pi$. Further increase of $\theta$ causes a rapid increase in the peak radius, which diverges as $\theta$ approaches $1.50561869 \pi$ and $\sigma(\theta)$ approaches $180^{\circ}$. In the narrow second band, the peak radius has a minimum value where $\theta$ and $\sigma$ are very close to $1.5 \pi$ radians (270 degrees).

The peak radius at the center of any band (approximately the minimum peak radius) is found by setting $\theta=\theta_{n}$ :

$$
a_{+\max }^{2}\left(\theta_{n}\right)=\in L \frac{e^{\theta_{n}}}{\theta_{n}}
$$

where $\theta_{n}$ is given by Eq. (30). From this, one finds that the minimum peak radius in the second band is about 2.78 times larger than in the first band and that it increases almost exponentially for larger $n$.

Figure 6 shows $a_{\max }$ for the first two bands in terms of the phase advance $\sigma$ rather than the field-strength parameter $\theta$ used for Fig. 5. As mentioned at the end of Sec. V, all the passbands have $\sigma$ width $180^{\circ}$. The stop bands described in Ref. [10] shrink, in the absence of space charge, to points at $180^{\circ}, 360^{\circ}$, etc.
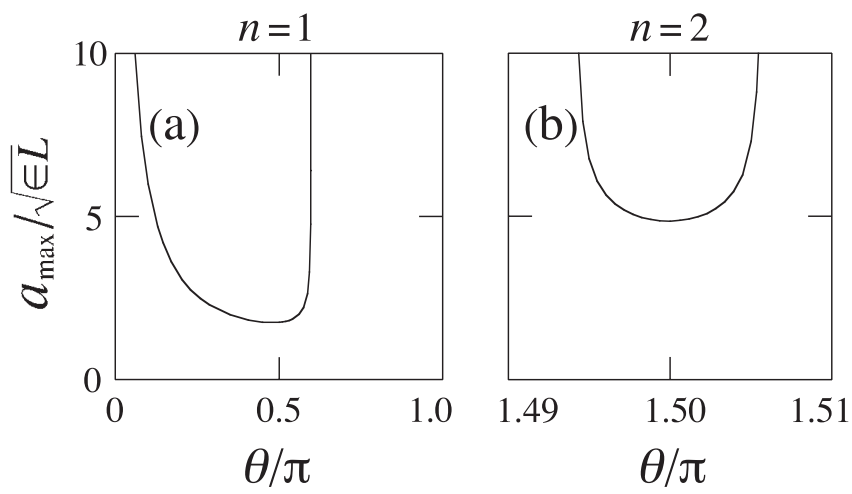

FIG. 5. (a) Values of $a_{\max } / \sqrt{\in L}$ [Eq. (36)] for the first stable band. (b) The second band with the $\theta$ axis magnified. The smallest possible envelope excursions occur for $\theta=0.47784 \pi$.

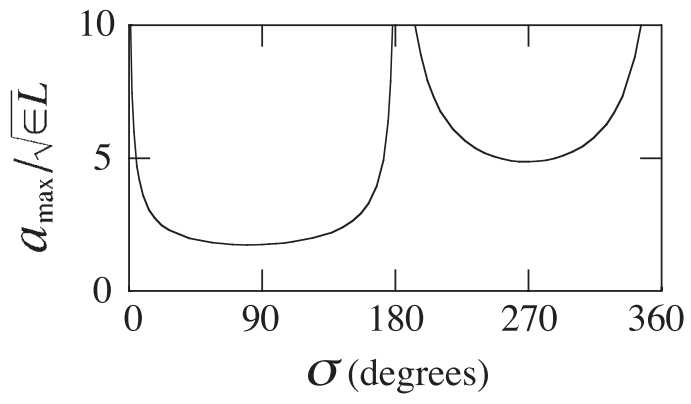

FIG. 6. Values of $a_{\max } / \sqrt{\in L}$ [Eq. (36)] for the first two stable bands as a function of phase advance $\sigma$ [Eq. (34)]. Minimum beam size occurs at $\sigma(\theta)=80.57^{\circ}$ but exceeds the minimum by less than $1 \%$ over the range $69^{\circ}$ to $93^{\circ}$.

\section{PHASE ADVANCE AS A FUNCTION OF $z$ FOR PASSBAND MIDPOINTS}

At the midpoints $\theta_{n}$, the cosine factor containing $z$ in Eq. (28) becomes

$$
\cos [\theta(2 z / L-1)]=\sin \left(\theta_{n} 2 z / L\right)
$$

and the denominators become $\mathrm{P} \theta$. Thus, $a^{2}$ at the midpoint of any band $n$ is

$a_{n+}{ }^{2}(n ; z)=\in L \frac{\cosh \theta_{n}+\sinh \theta_{n} \mathrm{P} \sin \left(\theta_{n} 2 z / L\right)}{\theta_{n}}$

$a_{n-}{ }^{2}(n ; z)=\frac{\in L}{\theta_{n}} \cosh \left[\theta_{n}(2 z / L-3)\right]$.

The reciprocals of Eqs. (39) are easily integrated (using appropriate branch selection), yielding the exact phase advance $\sigma(\theta ; z)$ along the $z$ axis:

$$
\sigma(\theta ; z)=\in \int_{0}^{z} \frac{d z^{\prime}}{a^{2}}
$$

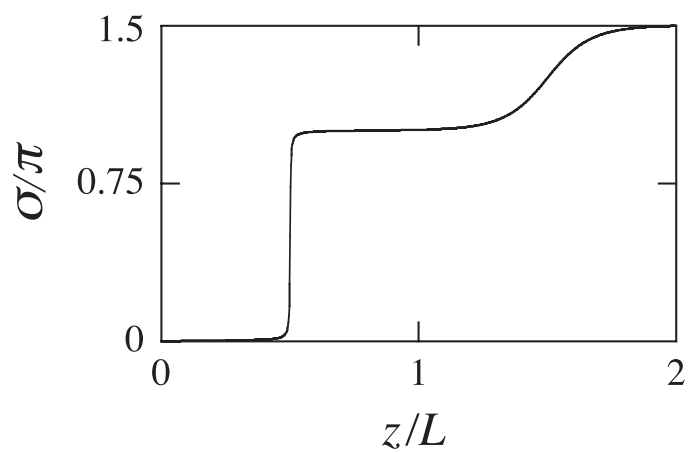

FIG. 7. Phase $\sigma$ as a function of $z$ for the center of the second stable band, from Eq. (41). Note the phase jump at $z=L / 2$. 
where the $\theta$ dependence enters through $a$. The integral is done in sections. We illustrate for band 2 , where $\theta_{2}=\frac{3 \pi}{2}$ :

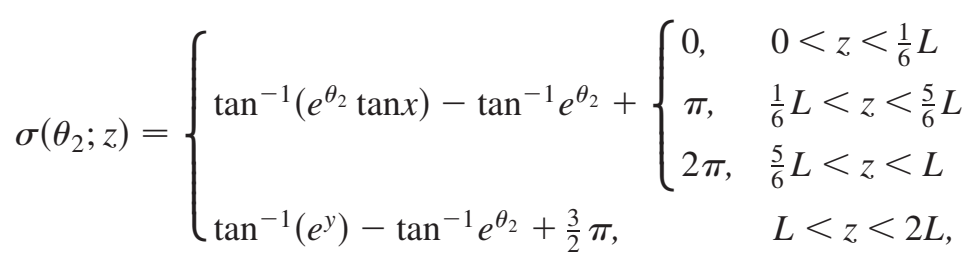

where

$$
x \equiv \frac{1}{2} \theta_{2}\left(\frac{2 z}{L}-1\right), \quad y \equiv \theta_{2}\left(\frac{2 z}{L}-3\right)
$$

and where the principal value of $\tan ^{-1}$ is used. The phase advance over a full period $(0,2 L)$ is $3 \pi / 2$, which agrees with Eq. (34) for $\theta_{2}$. The above result is plotted in Fig. 7.

\section{SECOND-BAND BEAM COMPRESSION}

Figure 3 showed that, in the second band, the beam radius is small in both the $x$ and $y$ directions for $z / L=$ 0.5 and for $z / L=1.5$ so that the beam area is highly compressed at these points. [Recall that $b(z)=a(z+L)$ for our matched beam.] The beam area is plotted on linear and $\log$ scales for the center (Fig. 8) and the edge (Fig. 9) of the second band. The KV equation predicts that the compression ratio is more than 200 in the former case and more than $10^{7}$ in the latter. However, various effects not taken into account by the CSS model will limit the beam compression. These and other practical matters are discussed in Sec. X.
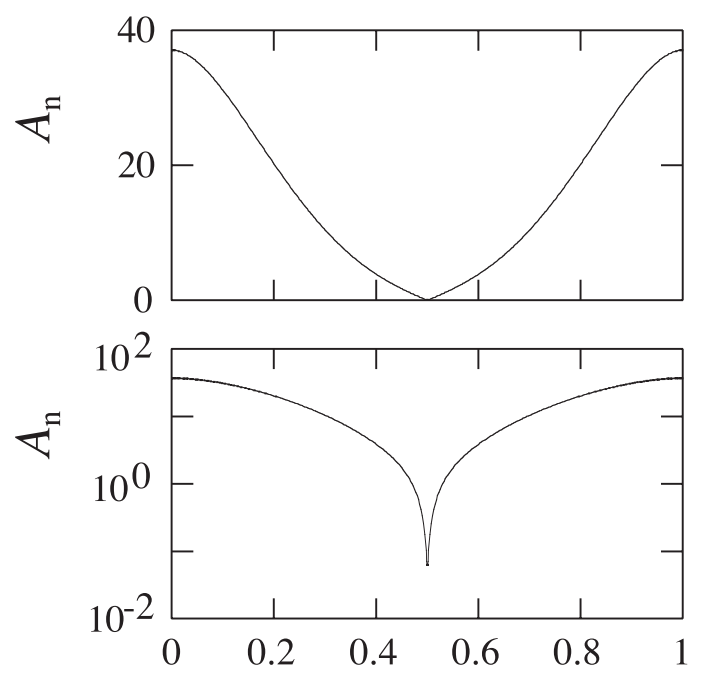

FIG. 8. Normalized beam area $A_{n} \equiv \pi a(z) a(z+L) / \in L[$ from Eqs. (28)] as a function of $z$ for the center of the second band. Focus-strength parameter $\theta=1.5 \pi, \sigma=270^{\circ}$ [cf. Fig. 3(c)]. The same curves apply to the range $L<z<2 L$.

\section{BEAM MATCHING EQUATION}

We now return to Eq. (28a) and restrict $z$ to a constant value, $z_{0}$. For the case $z_{0}=0$, with $a(0) \equiv a_{0}$,

$$
a_{0}^{2}=\in L \frac{s n c h+s h c s}{\mathrm{P} \theta \sqrt{1-c s^{2} c h^{2}}} .
$$

This is an exact matching condition for the CSS model which relates the initial beam amplitude and the parameters $\theta, \in$, and $L$. It holds for all values of $\theta$ and $\sigma$ except integer multiples of $\pi$. In the limit $\theta \rightarrow 0, a_{0} \rightarrow\langle a(z)\rangle$ and Eq. (42) reduces to the usual smooth-approximation matching condition $[3,4,6]$. For the CSS model, it can be written as $\langle a\rangle^{2} \rightarrow 2 \sqrt{3} \in L / \theta^{2}$.

It may be interesting to regard the exact matching equation (42) from another point of view. We observe that Eq. (4), together with periodic $K$ and the requirement that the beam be matched to the lattice, is a homogeneous system with periodic boundary conditions. This allows us to interpret the solution of the envelope equations for matched beams as an eigenvalue problem. The matching condition is then interpreted as the (nonlinear) equation for the eigenvalues. As typically occurs for nonlinear problems, the eigenfunction normalization, of which $a_{0}$ is a measure, enters the eigenvalue equation.
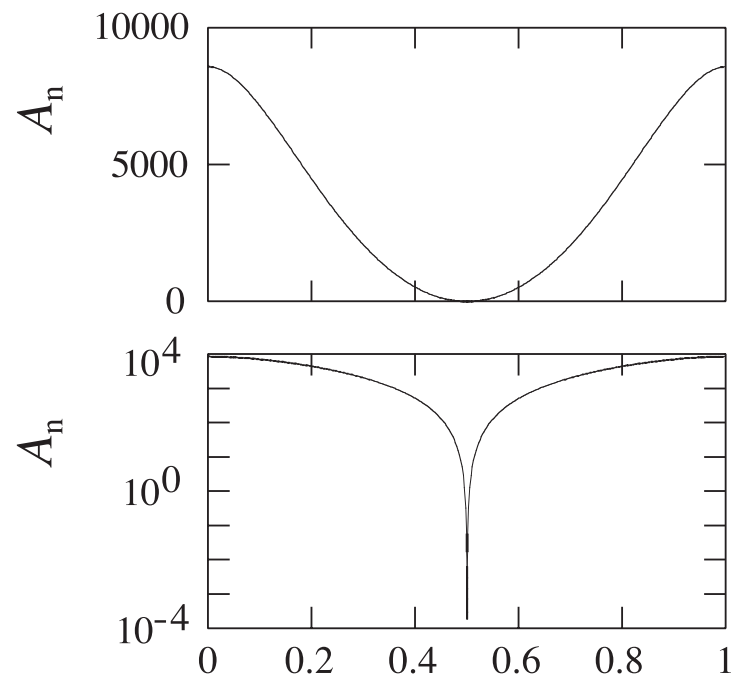

FIG. 9. Normalized beam area $A_{n} \equiv \pi a(z) a(z+L) / \in L[$ from Eqs. (28)] as a function of $z$ near the edge of the second band. Focus-strength parameter $\theta=1.50561868 \pi, \sigma=359.75^{\circ}$ [cf. Fig. 3(d)]. The same curves apply to the range $L<z<2 L$. 
We illustrate the above remarks using Eq. (36), which may be regarded as the matching equation for $a_{\max }$. From the alternative point of view, it is a transcendental equation for $\theta$, the eigenvalue, with measure $a_{\max }$. Some modes of its spectrum are indicated graphically in Fig. 5. One sees, for example, that the ordinate value $a_{\max } / \sqrt{\in L}=5$ intersects the plot twice in each of the first two bands and has no intersections in higher bands. There is a total of four modes in this example. Physically, this means that there are four different focusing strengths that give this particular value for $a_{\max } / \sqrt{\in L}$.

\section{DISCUSSION OF THE SECOND BAND}

For charge-dominated beams, some authors, e.g. [14], have recommended operating in higher bands to transport larger beam current. Lund has pointed out that the envelope excursions will then increase [10]. Emittance-dominated beams also exhibit this excursion increase, as shown by Eq. (37). For a given aperture, the particle flux is reduced in the higher bands. Nevertheless, there may be some uses for the second band, assuming such operation to be feasible in practice.

In the emittance-dominated case, the second band has a special feature: The beam radii in both planes can be small at the same points, as noted in Fig. 3. The series of localized regions of high compression (Sec. VIII) has possible applications such as differential pumping of the beam line using diaphragms. A truncated lattice could produce an extremely dense final focal spot, which, if achievable, would have various applications-for example, heating or compression of targets, which might be useful for warm dense matter studies.

The design of a machine for second-band operation would face formidable difficulties. Compared with the focusing field for $\sigma=90^{\circ}$, the field for $270^{\circ}$ is 9 times stronger. The narrowness in $\theta$ of the second band would require accurate field regulation-increasingly accurate as the band edge is approached. Obviously, the case of Fig. 9 is far beyond practical reach. It would be difficult to launch a beam in the second band. Launching at $z=0$, for example, would require steeply converging and diverging envelope angles in the two symmetry planes. All these would be technical challenges.

Effects have been neglected in this paper that would reduce the beam compression. Large beam excursions will produce focusing aberrations, not treatable by the $\mathrm{KV}$ equations with their paraxial approximation. As mentioned above, significant space-charge fields will widen the focus.
(However, this may be mitigated since emittance pressure eventually dominates as the beam is compressed.) All these effects remain to be studied.

A final remark applies to any band: The full KV equations, which include space-charge terms, require the KV distribution function for the beam [13] in order to produce the linear transverse self-field required for a periodic solution. The beam density, then, is uniform with a sharp cutoff at the boundary. On the other hand, low-space-charge models such as the CSS model allow periodic solutions to be obtained with distribution functions that yield realistic density profiles.

\section{ACKNOWLEDGMENTS}

We thank S. M. Lund for many useful comments and editing help, E. P. Lee for suggestions on an early version, and the referees for improving the final product. This work was supported in part by the U.S. Department of Energy under Contract No. DE-AC02-05CH11231.

[1] I. M. Kapchinskij and V. V. Vladimirskij, in International Conference on High Energy Accelerators and Instrumentation (CERN Scientific Information Service, Geneva, 1959), p. 274.

[2] S. M. Lund, S. H. Chilton, and E. P. Lee, Phys. Rev. ST Accel. Beams 9, 064201 (2006).

[3] M. Reiser, Part. Accel. 8, 167 (1978).

[4] R. C. Davidson, Physics of Nonneutral Plasmas (AddisonWesley, New York, 1990).

[5] E. P. Lee, Part. Accel. 52, 115 (1996).

[6] O. A. Anderson, Phys. Rev. ST Accel. Beams 10, 034202 (2007).

[7] E. D. Courant and H. S. Snyder, Ann. Phys. (Paris) 3, 1 (1958).

[8] Reference [7], pp. 2 and 15, uses "CLS" to refer to an earlier paper by Courant, Livingston, and Snyder.

[9] K. Takayama, Part. Accel. 21, 259 (1987).

[10] S. M. Lund and B. Bukh, Phys. Rev. ST Accel. Beams 7, 024801 (2004).

[11] "CSS" stands for "Courant-Snyder, Straight axis".

[12] S. Humphries, Jr., Principles of Charged Particle Acceleration (Wiley, New York, 1986), free online at http://www.fieldp.com.

[13] H. Wiedemann, Particle Accelerator Physics (SpringerVerlag, New York, 1993).

[14] R. Pakter and F.B. Rizzato, Phys. Rev. E 65, 056503 (2002). 